\title{
EXOSAT X-ray spectra of quasars
}

\section{Citation}

Comastri, Andrea, Giancarlo Setti, Giovanni Zamorani, Martin Elvis, Belinda J. Wilkes, Jonathan C. McDowell, and Paolo Giommi. 1992. "EXOSAT X-Ray Spectra of Quasars." The Astrophysical Journal 384 (January): 62. doi:10.1086/170851.

\section{Published Version}

doi:10.1086/170851

\section{Permanent link}

http://nrs.harvard.edu/urn-3:HUL.InstRepos:30212144

\section{Terms of Use}

This article was downloaded from Harvard University's DASH repository, and is made available under the terms and conditions applicable to Other Posted Material, as set forth at http:// nrs.harvard.edu/urn-3:HUL.InstRepos:dash.current.terms-of-use\#LAA

\section{Share Your Story}

The Harvard community has made this article openly available.

Please share how this access benefits you. Submit a story.

Accessibility 
THE ASTROPHYSiCAL JoURNAL, 384:62-71, 1992 January 1

(C) 1992. The American Astronomical Society. All rights reserved. Printed in U.S.A.

\author{
EXOSAT X-RAY SPECTRA OF QUASARS \\ Andrea Comastri, ${ }^{1}$ Giancarlo Setti, ${ }^{2}$ Giovanni Zamorani, ${ }^{3}$ Martin Elvis, ${ }^{4}$ Paolo Giommi, ${ }^{5}$ \\ Belinda J. Wilkes, ${ }^{4}$ AND Jonathan C. MCDOWELl ${ }^{4}$ \\ Received 1991 February 28; accepted 1991 July 5
}

\begin{abstract}
We have measured the X-ray spectra in the $0.1-10 \mathrm{keV}$ energy range for 17 AGNs (mostly PG quasars) using the EXOSAT Low and Medium Energy arrays. The sample has been selected on the basis of soft X-ray flux and includes all the AGNs in the 1981 Zamorani et al. and 1986 Tananbaum et al. publications with an Einstein flux greater than $6 \times 10^{-12}$ ergs $\mathrm{cm}^{-2} \mathrm{~s}^{-1}$.

For each object we have determined the best-fit spectral parameters in the medium-energy range $(\sim 2-10$ $\mathrm{keV}$ ), i.e., the power-law slope, the normalization, and the absorbing column density. In this energy range, all the spectra are well described by a single power law model, with a wide distribution of energy spectral indices in the range $0.4\langle\alpha<1.3$. The average spectral index is $\langle\alpha\rangle=0.89 \pm 0.06$, but most of the objects are distributed around $\alpha \simeq 1.0$. The overall distribution of spectral indices is significantly displaced toward steeper spectra, when compared to that found in the same energy band by Turner \& Pounds, for a large sample of hard X-ray-selected Seyfert galaxies. This difference can be understood in terms of the different selection criteria of the two samples (soft versus hard X-ray selection), and it is concluded that the true distribution of hard X-ray spectral indices in AGNs is probably wider than previously suggested.

Inclusion of lower energy data $(\sim 0.1-2.0 \mathrm{keV})$ shows the presence of significant soft excess in six out of 17 cases. This soft excess emission can be modeled by either a steep power law with $\alpha$ a ranging from 2.7 to 4.2 or the high-energy tail of a blackbody with temperatures in the range 40-80 eV. The intersection of this soft excess emission with the hard X-ray power law is in the energy interval $0.35-0.75 \mathrm{keV}$, in the source rest frame.

A correlation analysis performed on our data set does not reveal any correlation between the mediumenergy spectral properties and other physical parameters of the sources. However, fits performed in the energy range 0.1-4.0 keV (comparable with the Einstein IPC energy range) show a significant correlation between radio-loudness and $\mathrm{X}$-ray spectral properties, with the radio-loud objects having, on average, flatter slopes than the radio-quiet ones, in agreement with the findings of Wilkes \& Elvis. The fact that the same correlation is not seen in our data at higher energy seems to be explained by a correlation between presence and strength of soft excess and "radio-quietness": the objects with significant soft excess emission tend to be the most radio-quiet quasars in our sample.
\end{abstract}

Subject headings: galaxies: nuclei — galaxies: Seyfert — quasars: general - X-rays: galaxies

\section{INTRODUCTION}

Quasars and Seyfert 1 galaxies are well known to be powerful sources of X-rays which constitute an important, and sometimes even dominant, fraction of their total energy output. Although the nature of the X-ray emission is still uncertain, it is widely believed that it might be associated with the activity induced by a central compact object, perhaps a massive black hole. The study of the X-ray spectra together with an intercomparison of the spectral behavior in other portions of the emitted radiation spectrum, may provide important clues to

\footnotetext{
${ }^{1}$ Dipartimento di Astronomia, Università di Bologna, via Zamboni 33, 40126 Bologna, Italy; also Istituto di Radioastronomia del CNR, Bologna, Italy.

${ }^{2}$ Istituto di Radioastronomia del CNR, via Irnerio 46, 40126 Bologna, Italy; also Dipartimento di Astronomia, Università di Bologna and European Southern Observatory, Garching bei München, Germany.

${ }^{3}$ Osservatorio Astronomico di Bologna, via Zamboni 33, 40126 Bologna, Italy; also Istituto di Radioastronomia del CNR, Bologna, Italy.

${ }^{4}$ Harvard-Smithsonian Center for Astrophysics, 60 Garden Street, Cambridge, MA 02138 .

${ }^{5}$ EXOSAT Observatory, Astrophysics Division, Space Science Department of ESA; postal address: Code SAE, ESTEC, Postbus 229, 2200 AG Noordwijk, The Netherlands. On leave of absence from Istituto di Fisica Cosmica del CNR, Milano.
}

understanding the nature of the emission mechanisms and the physical conditions of the regions surrounding the central compact object. In addition, the study of the X-ray spectra of quasars and Seyfert 1 galaxies is important to set constraints to the integrated contribution of these objects to the extragalactic $\mathrm{X}$-ray background (XRB), whose origin is still the subject of debate (see Setti 1991 for a recent discussion).

Early surveys in the $2-20 \mathrm{keV}$ range of X-ray-bright AGNs (mostly low-luminosity and low-redshift Seyfert 1 galaxies) suggested the existence of a "universal" energy spectrum described by a power law of the form $S(E) \propto E^{-\alpha}$, where $E$ is the photon energy and $\alpha \simeq 0.7$. The small spread in the observed distribution of the spectral indices was consistent with the expected dispersion introduced by the observational uncertainties only (Mushotzky 1984). It should be noted that the results obtained from the Japanese mission Ginga show that the spectra of a large fraction of low-luminosity AGNs flatten at $\gtrsim 10 \mathrm{keV}$. Turner \& Pounds $(1989$, hereafter TP89) reported EXOSAT observations of a larger sample of 48 hard X-ray-selected AGNs (again mostly Seyfert 1 nuclei) and confirmed that the energy spectra in the $2-10 \mathrm{keV}$ range are adequately described by simple power laws, with a narrow distribution (dispersion $\sigma=0.16$ ) of spectral indices around a mean energy index of about 0.7 . 
Ginga measurements of the spectra $(2-10 \mathrm{keV})$ of a small sample of quasars (five radio-loud and three radio-quiet) give a mean spectral slope of 0.69 , but with a relatively high dispersion of about 0.29 (Turner et al. 1989). For this sample, no correlation between radio-loudness and the $2-10 \mathrm{keV}$ spectral slope is found.

The X-ray spectra of a sample of 20 Seyfert 1 galaxies observed over a broader energy range $(\sim 0.15-10 \mathrm{keV}$; IPC and MPC instruments of the Einstein Observatory) show the "canonical" average energy index of 0.7 at energies $\geq 2 \mathrm{keV}$ (Urry et al. 1989). From the combined IPC/MPC data, it is concluded that, although a single power-law model is generally acceptable, in most cases a power law plus a soft excess emission modeled by a blackbody give a significantly better description of the data.

In the soft-X-ray domain there are at least two effects which complicate the description of the X-ray spectra. The first, intrinsic absorption, has been shown to be important mainly in low-luminosity AGNs (Reichert et al. 1985; Lawrence \& Elvis 1982). The second, soft excess, was quite clearly seen in the study of the Einstein IPC data of a sample of 33 quasars (Wilkes \& Elvis 1987, hereafter WE87) and then confirmed to be a common feature in EXOSAT data of Seyfert galaxies (TP89). The Einstein IPC data (0.3-3.5 keV) showed (Elvis et al. 1986; WE87) that, although the soft X-ray spectra of AGNs have a wide range of power-law spectral indices $(-0.2<\alpha<1.8)$, they are strongly grouped around $\alpha \sim 0.5$ for radio-loud objects and $\alpha \sim 1.0$ for radio-quiet objects. This difference has been statistically confirmed from the analysis of a larger sample of quasars, covering a wide redshift range (Canizares \& White 1989).

A recent analysis of Einstein IPC observations of a large sample of mostly radio-quiet Seyfert 1 galaxies (75 objects) shows a mean spectral index $\alpha=0.81 \pm 0.03$, significantly steeper than the "universal" hard energy slope (Kruper, Urry, \& Canizares 1990). Although the number of radio-loud objects in this sample is small, the results are consistent with the correlation seen in quasars between soft spectral indices and radio loudness.

Elvis et al. (1986) discussed various possibilities which may explain the observed difference in the spectral index distributions between the IPC results and those obtained at harder energies. They concluded that, while the lower energy range of the IPC is relevant in a fraction of the objects, the most likely explanation for the observed difference is biases induced by the selection of the samples to be compared. To further investigate this effect we have used EXOSAT Low and Medium Energy data to determine the energy spectral index of a number of AGNs, selected on the basis of soft (i.e., Einstein) X-ray flux.

\section{OBSERVATIONS AND ANALYSIS}

We present new X-ray data for a sample of X-ray-bright Seyfert 1 nuclei and quasars (hereafter AGNs) observed with the EXOSAT Observatory. The objects in the sample were selected on the basis of soft (Einstein) X-ray flux. In particular, the sample includes all the AGNs in the publications of Zamorani et al. (1981) and Tananbaum et al. (1986) with an Einstein flux greater than $6 \times 10^{-12} \mathrm{ergs} \mathrm{cm}^{-2} \mathrm{~s}^{-1}$, or equivalently 0.2 IPC counts $\mathrm{s}^{-1}$, contained in the EXOSAT data base as of 1990 May. A total number of 17 AGNs satisfy this selection criterion; most of them are PG quasars (Schmidt \& Green 1983). Table 1 lists some relevant data for these objects. $H_{0}=$ $50 \mathrm{~km} \mathrm{~s}^{-1} \mathrm{Mpc}^{-1}$ and $q_{0}=0.0$ are used throughout this paper.

TABLE 1

THE SAMPLE

\begin{tabular}{|c|c|c|c|c|c|c|c|c|}
\hline $\begin{array}{l}\text { Coordinate } \\
\text { (1) }\end{array}$ & $\begin{array}{l}\text { Name } \\
(2)\end{array}$ & $\begin{array}{l}\text { Redshift } \\
\text { (3) }\end{array}$ & $\begin{array}{l}M_{B}^{\mathrm{a}} \\
(4)\end{array}$ & $\begin{array}{l}S_{X}^{\mathrm{b}} \\
(5)\end{array}$ & $\begin{array}{l}L_{X}{ }^{\mathrm{c}} \\
(6)\end{array}$ & $\begin{array}{c}\alpha_{\mathrm{IPC}}{ }^{\mathrm{d}} \\
(7)\end{array}$ & $\begin{array}{l}\alpha_{o x}^{e} \\
(8)\end{array}$ & $\begin{array}{l}R_{L}{ }^{\mathrm{f}} \\
(9)\end{array}$ \\
\hline $0003+199 \ldots \ldots \ldots \ldots$ & PG, Mrk 335 & 0.025 & -22.55 & 76.6 & 2.0 & $\ldots$ & 1.25 & 0.55 \\
\hline $0007+106 \ldots \ldots \ldots \ldots$ & PG, III Zw 2 & 0.089 & -22.93 & 19.0 & 9.0 & $0.4_{-0.4}^{+1.2}$ & 1.06 & 2.24 \\
\hline $0026+129 \ldots \ldots \ldots \ldots$ & PG & 0.142 & -25.13 & 7.0 & 7.6 & $0.9_{-0.3}^{+0.3}$ & 1.43 & 0.34 \\
\hline $0049+171 \ldots \ldots \ldots \ldots$ & PG, Mrk 1148 & 0.064 & -22.45 & 9.0 & 2.0 & $0.7_{-0.4}^{+3.0}$ & 1.24 & -0.49 \\
\hline $0052+251 \ldots \ldots \ldots \ldots$ & PG & 0.155 & -24.95 & 7.1 & 9.5 & $1.1_{-0.7}^{+2.3}$ & 1.36 & -0.61 \\
\hline $0637-752 \ldots \ldots \ldots \ldots$ & PKS & 0.651 & -27.65 & 6.9 & 230.0 & $0.5_{-0.4}^{+0.9}$ & 1.25 & 3.52 \\
\hline $0804+761 \ldots \ldots \ldots \ldots$ & PG & 0.100 & -24.30 & 10.9 & 5.8 & $\ldots$ & 1.34 & -0.21 \\
\hline $0923+129 \ldots \ldots \ldots \ldots$ & PG, Mrk 705 & 0.029 & -21.70 & 8.2 & 0.4 & $\ldots$ & 1.41 & 0.32 \\
\hline $1211+143 \ldots \ldots \ldots \ldots$ & PG & 0.085 & -24.25 & 32.3 & 11.8 & $1.8_{-0.4}^{+0.5}$ & 1.22 & -0.72 \\
\hline $1217+023 \ldots \ldots \ldots \ldots$ & PKS & 0.240 & -24.60 & 8.6 & 26.0 & $0.5_{-0.2}^{+0.5}$ & 1.14 & 2.38 \\
\hline $1219+756 \ldots \ldots \ldots \ldots$ & Mrk 205 & 0.070 & -22.95 & 17.0 & 2.9 & $0.8_{-0.2}^{+0.2}$ & 1.25 & -0.31 \\
\hline $1226+023 \ldots \ldots \ldots \ldots$ & PG, 3C 273 & 0.158 & -27.43 & 83.4 & 117.0 & $0.3_{-0.1}^{+0.2}$ & 1.32 & 3.06 \\
\hline $1229+204 \ldots \ldots \ldots \ldots$ & PG, Ton 1542 & 0.064 & -23.58 & 6.5 & 1.5 & $\ldots$ & 1.47 & -0.96 \\
\hline $1426+015 \ldots \ldots \ldots \ldots$ & PG, Mrk 1383 & 0.086 & -23.90 & 16.0 & 6.7 & $0.9_{-0.2}^{+0.2}$ & 1.26 & -0.55 \\
\hline $1501+106 \ldots \ldots \ldots \ldots$ & PG, Mrk 841 & 0.036 & -21.93 & 28.5 & 2.0 & $1.0_{-0.4}^{+0.2}$ & 1.16 & -0.67 \\
\hline $2130+099 \ldots \ldots \ldots \ldots$ & PG, II Zw 136 & 0.061 & -23.75 & 8.8 & 1.8 & $0.8_{-0.4}^{+0.4}$ & 1.46 & 0.37 \\
\hline $2135-147 \ldots \ldots \ldots \ldots$ & PKS, PHL 1657 & 0.200 & -25.18 & 7.1 & 17.0 & $0.5_{-0.2}^{+0.4}$ & 1.30 & 1.70 \\
\hline
\end{tabular}

a Absolute $B$ magnitude.

b X-ray flux (in units of $10^{-12} \mathrm{ergs}^{-2} \mathrm{~cm}^{-1}$ ) in the energy range 0.3-3.5 keV (Zamorani et al. 1981 and Tananbaum et al. 1986).

${ }^{c} \mathrm{X}$-ray luminosity (in units of $10^{44} \mathrm{ergs} \mathrm{s}^{-1}$ ) in the energy range 0.5-4.5 keV (Zamorani et al. 1981 and Tananbaum et al. 1986).

${ }^{\mathrm{d}}$ IPC spectral slope taken from Wilkes \& Elvis 1987, except for PG 0049+171 (Elvis et al. 1986) and PG $0052+251$ (Elvis et al. 1991).

e Nominal slope between optical and X-ray bands defined in Zamorani et al. 1981.

f Radio-loudness index taken from Wilkes \& Elvis 1987, when available, or computed from Kellerman et al. 1989 data. 
For all the objects in our sample the EXOSAT data were obtained with both the low-energy (LE) imaging telescopes (De Korte et al. 1981) and the medium-energy (ME) detector array I (Turner, Smith, \& Zimmerman 1981). Although the ME detector was in principle sensitive up to about $50 \mathrm{keV}$, the signal-tonoise ratio becomes very poor above $\sim 8 \mathrm{keV}$ for the relatively weak sources of our sample.

Table 2 gives the observing log. When more than one observation was available in the EXOSAT data base for the same objects, we have used the data set with the longer exposure time and/or higher quality. Most of the objects have been observed with at least two low-energy filters (3000 Lexan and $\mathrm{Al} / \mathrm{P}$ ), the only exception being PKS 0637-75 for which there is only one observation with the Lexan filter. For 10 objects, additional LE data with the boron filter are also available, while for one object (PG 1229+204) we have also one observation with the PPL filter. For all filters (with the exception of the boron) we have used vignetting corrected and background subtracted data from the EXOSAT data base. The boron case is special since the point-spread function in this filter strongly depends on the source spectral shape. For this reason a careful reanalysis of all the original image data has been performed using the interactive analysis system of the EXOSAT Observatory. While the boron filter has a narrow energy response peaked at around $1 \mathrm{keV}$, the Lexan and $\mathrm{Al} / \mathrm{P}$ filters are still sensitive at much lower energies, well below the $C$ band at 0.28 keV (White \& Peacock 1988).

The combination of the count rates in low-energy filters with the more detailed spectral information given by the mediumenergy detector at higher energies not only provides a better determination of the spectral slope but also allows to constrain the degree of interstellar and/or intrinsic absorption and to detect, if present, additional soft components.

\subsection{ME Spectral Fitting}

For all our objects, the ME analysis has been performed only in the energy range where a net signal above the background was detected. The highest PHA channel used ranges from channel 25 , corresponding to about $7 \mathrm{keV}$, to channel 35 (about 9-10 keV). For each object, the pulse-height spectrum was fitted with a source power law spectrum corrected for absorption due to interstellar gas of solar composition (Morrison \& McCammon 1983). For the fitting procedure we used the standard $\chi^{2}$ minimization technique available in the EXOSAT spectral fitting package (XSPEC; Shafer, Haberl, \& Arnaud 1989).

In a first fitting run, both the slope of the power-law spectrum and the amount of absorbing hydrogen were considered as free parameters and the $90 \%$ confidence limits on each parameter were calculated (Avni 1976; Lampton, Margon, \& Bowyer 1976). Because of the relative faintness of our objects, with typical fluxes in the range 4 to $8 \times 10^{-12} \mathrm{ergs} \mathrm{cm}^{-2} \mathrm{~s}^{-1}$, the allowed region in the $N_{\mathrm{H}^{-}} \alpha$ plane is almost always very large. Since the values of Galactic $N_{\mathrm{H}}$, excepting only PG $0049+171$, are well inside the allowed regions and mostly very close to the best-fit values, we have decided to $\operatorname{keep} N_{\mathrm{H}}$ fixed at the Galactic values. The results of these fits are shown in Table 3. The simple power-law model with absorption from our own Galaxy provides an acceptable fit for essentially all the objects (reduced $\chi^{2}$ of the order of unity). The overall distribution of the reduced $\chi^{2}$ for the entire sample (with $N_{\mathrm{H}}=N_{\mathrm{H}_{\mathrm{gal}}}$ ) is consistent with that expected by chance according to a KolmogorovSmirnov test $(p \sim 0.2)$. Therefore our data do not indicate the presence of any substantial amount of intrinsic absorbing cold matter. PG $0049+171$, with a reduced $\chi^{2}$ of $1.98(17$ degrees of freedom), appears to be the only object for which a substantial $N_{\mathrm{H}}$ in excess of Galactic $N_{\mathrm{H}}$ seems to be required (see footnote $\mathrm{f}$, Table 3). Inspection of the data for this object shows that an important contribution to the large $\chi^{2}$ is due to a single channel (channel 15 at about $4.2 \mathrm{keV}$ ) which is significantly higher than the adjacent channels. Excluding this "peculiar" channel from the fit, the reduced $\chi^{2}$ with $N_{\mathrm{H}}=N_{\mathrm{H}_{\mathrm{gal}}}$ becomes 1.31. Thermal bremsstrahlung models were also tried, but they generally provided significantly worse fits to the data.

\subsection{LE and $M E$ Combined Spectral Fitting}

A single power-law plus cold gas absorption model was then fitted to the combined LE and ME data. The results of these fits are shown in Table 4, where for each object we give two sets of results, the first with both the spectral slope (col. [2]) and the amount of $N_{\mathrm{H}}$ (col. [3]) considered as free parameters and the second with $N_{\mathrm{H}}$ kept fixed at the Galactic value.

In order to check whether the addition of the LE data is consistent with the model of a single power law, we have applied the $F$-test, i.e., for each object we have computed the $F$-statistics (Bevington 1969): $F\left(\chi^{2}, v_{1}, v_{2}\right)=\left(\Delta \chi^{2} / \Delta v_{1}\right) /\left(\chi_{\mathrm{ME}}^{2} / v_{2}\right)$, where $\Delta v_{1}=$ number of additional data points (i.e., number of LE filters) and $v_{2}=$ number of degrees of freedom in the ME filters. Adopting as a critical value for $F$ the value corresponding to the probability of $5 \%$, we find that for six objects (all of them $P G$ quasars: $0003+199,1211+143,1226+023$, $1229+204,1426+015$, and $1501+106)$ the $F$-value in the case $N_{\mathrm{H}}=N_{\mathrm{H}_{\text {gal }}}$ is larger than $F_{\text {crit }}\left(F_{\text {crit }} \sim 3\right.$ for the relevant number of degrees of freedom). This suggests that for these objects, a single power-law model is not an acceptable description of the spectrum over the energy range $0.1-8 \mathrm{keV}$. For all these objects the best-fit value for $N_{\mathrm{H}}$ is significantly smaller than the measured value of $N_{\mathrm{H}_{\mathrm{gal}}}$ and, moreover, for all of them the best-fit slope (at $N_{\mathrm{H}}=N_{\mathrm{H}_{\mathrm{gal}}}$ ) of the $\mathrm{LE}+\mathrm{ME}$ data is steeper than the corresponding slope of ME data only. On the basis of these independent checks, we conclude that we have detected the presence of a soft excess in these six objects for which a more detailed spectral analysis is reported below.

For the other 11 objects the $F$-value for $N_{\mathrm{H}}=N_{\mathrm{H}_{\text {gal }}}$ is well below the value of $F_{\text {crit }}$ and $N_{\mathrm{H}_{\mathrm{gal}}}$ is always within the allowed $N_{\mathrm{H}}$ range. This is true also for PG $0049+171$, for which the ME data seemed to require a substantial amount of $N_{H}\left(>10^{22}\right.$ $\mathrm{cm}^{-2}$ ). In light of the possible problems with at least one $\mathrm{ME}$ channel for this object (see $\S 2.1$ ) we tentatively conclude that the combined LE and ME data are consistent with a single power-law spectrum over the entire energy range $0.1-8 \mathrm{keV}$, with no significant absorption in addition to the Galactic one.

For the six objects classified as "soft excess" in Table 4, we have tried more complex fits to the data. In Table 5 we report the best-fit results for two models, one described by two power laws and the other by a blackbody plus a power law with the high-energy slope and normalization fixed at the value found from the ME fit. In order to find a lower limit for the strength of the soft excess emission, we have fixed the amount of $N_{\mathrm{H}}$ at the minimum possible value, i.e., $N_{\mathbf{H}}=N_{\mathrm{H}_{\mathrm{gal}}}$. To test the statistical significance of the models used to fit the soft excess emission, we have performed an $F$-test. The addition of two free parameters $\left(\alpha_{\text {steep }}\right.$ or the blackbody temperature with the relative normalizations) gives a statistically better fit for all six objects. 
TABLE 2

LOG OF OBSERVATIONS

\begin{tabular}{|c|c|c|c|c|}
\hline Object & Date & Detector & $\begin{array}{l}\text { Exposure Time } \\
\text { (s) }\end{array}$ & Count Rate ${ }^{a}$ \\
\hline $0003+199 \ldots \ldots \ldots \ldots$ & 1984 Dec 6 & $\begin{array}{l}\text { ME } \\
\text { 3lx } \\
\mathrm{Al} / \mathrm{P} \\
\text { Boron }\end{array}$ & $\begin{array}{r}32370 \\
4732 \\
5321 \\
19770\end{array}$ & $\begin{array}{r}1.25 \pm 0.03 \\
20.00 \pm 0.90 \\
9.39 \pm 0.56 \\
0.93 \pm 0.09\end{array}$ \\
\hline $0007+106 \ldots \ldots \ldots \ldots$ & 1985 Dec 20 & $\begin{array}{l}\text { ME } \\
31 \mathrm{x} \\
\mathrm{Al} / \mathrm{P}\end{array}$ & $\begin{array}{r}22870 \\
15671 \\
2291\end{array}$ & $\begin{array}{l}2.60 \pm 0.04 \\
3.50 \pm 0.20 \\
2.65 \pm 0.43\end{array}$ \\
\hline $0026+129 \ldots \ldots \ldots \ldots$ & 1984 Jul 16 & $\begin{array}{l}\mathrm{ME} \\
31 \mathrm{x} \\
\mathrm{Al} / \mathrm{P} \\
\text { Boron }\end{array}$ & $\begin{array}{r}34540 \\
5520 \\
5930 \\
21008\end{array}$ & $\begin{array}{l}0.51 \pm 0.04 \\
1.61 \pm 0.22 \\
1.16 \pm 0.18 \\
0.43 \pm 0.15\end{array}$ \\
\hline $0049+171 \ldots \ldots \ldots \ldots$ & 1985 Nov 30 & $\begin{array}{l}\mathrm{ME} \\
31 \mathrm{x} \\
\mathrm{Al} / \mathrm{P}\end{array}$ & $\begin{array}{r}20100 \\
18888 \\
3472\end{array}$ & $\begin{array}{l}0.71 \pm 0.04 \\
1.43 \pm 0.12 \\
0.82 \pm 0.21\end{array}$ \\
\hline $0052+251 \ldots \ldots \ldots \ldots$ & 1985 Dec 20 & $\begin{array}{l}\text { ME } \\
31 \mathrm{x} \\
\mathrm{Al} / \mathrm{P}\end{array}$ & $\begin{array}{r}22170 \\
10841 \\
5863\end{array}$ & $\begin{array}{l}0.30 \pm 0.04 \\
1.65 \pm 0.16 \\
0.92 \pm 0.17\end{array}$ \\
\hline $0637-752 \ldots \ldots \ldots \ldots$ & $1984 \operatorname{Sep} 8$ & $\begin{array}{l}\text { ME } \\
31 \mathrm{x}\end{array}$ & $\begin{array}{l}15030 \\
14340\end{array}$ & $\begin{array}{l}0.41 \pm 0.04 \\
0.50 \pm 0.08\end{array}$ \\
\hline $0804+761 \ldots \ldots \ldots \ldots$ & 1985 Mar 16 & $\begin{array}{l}\mathrm{ME} \\
\text { 31x } \\
\mathrm{Al} / \mathrm{P} \\
\text { Boron }\end{array}$ & $\begin{array}{r}26090 \\
4237 \\
5563 \\
10735\end{array}$ & $\begin{array}{l}0.81 \pm 0.03 \\
3.30 \pm 0.30 \\
2.50 \pm 0.30 \\
0.35 \pm 0.13\end{array}$ \\
\hline $0923+129 \ldots \ldots \ldots \ldots$ & 1985 May 9 & $\begin{array}{l}\mathrm{ME} \\
31 \mathrm{x} \\
\mathrm{Al} / \mathrm{P} \\
\text { Boron }\end{array}$ & $\begin{array}{r}12220 \\
3110 \\
6568 \\
13026\end{array}$ & $\begin{array}{l}1.02 \pm 0.08 \\
2.70 \pm 0.40 \\
1.80 \pm 0.20 \\
0.43 \pm 0.15\end{array}$ \\
\hline $1211+143 \ldots \ldots \ldots \ldots$ & 1985 Jun 13 & $\begin{array}{l}\text { ME } \\
\text { 31x } \\
\mathrm{Al} / \mathrm{P} \\
\text { Boron }\end{array}$ & $\begin{array}{r}24470 \\
1257 \\
3539 \\
16560\end{array}$ & $\begin{array}{r}0.88 \pm 0.04 \\
20.20 \pm 1.50 \\
8.80 \pm 0.62 \\
0.88 \pm 0.25\end{array}$ \\
\hline $1217+023 \ldots \ldots \ldots \ldots$ & 1984 Jan 29 & $\begin{array}{l}\text { ME } \\
31 \mathrm{x} \\
\mathrm{Al} / \mathrm{P}\end{array}$ & $\begin{array}{r}17880 \\
5052 \\
7968\end{array}$ & $\begin{array}{l}0.44 \pm 0.09 \\
2.54 \pm 0.28 \\
1.17 \pm 0.16\end{array}$ \\
\hline $1219+756 \ldots \ldots \ldots \ldots$ & 1984 Jan 27 & $\begin{array}{l}\mathrm{ME} \\
31 \mathbf{x} \\
\mathrm{Al} / \mathrm{P}\end{array}$ & $\begin{array}{l}9950 \\
1679 \\
5293\end{array}$ & $\begin{array}{l}0.57 \pm 0.07 \\
2.94 \pm 0.51 \\
1.34 \pm 0.21\end{array}$ \\
\hline $1226+023 \ldots \ldots \ldots \ldots$ & 1985 May 18 & $\begin{array}{l}\mathrm{ME} \\
\text { 31x } \\
\mathrm{Al} / \mathrm{P} \\
\text { Boron }\end{array}$ & $\begin{array}{r}22820 \\
5424 \\
3332 \\
10546\end{array}$ & $\begin{array}{r}6.27 \pm 0.04 \\
26.00 \pm 1.00 \\
13.30 \pm 0.82 \\
2.20 \pm 0.19\end{array}$ \\
\hline $1229+204 \ldots \ldots \ldots \ldots$ & 1985 Jun 8 & $\begin{array}{l}\mathrm{ME} \\
\mathrm{Al} / \mathrm{P} \\
\text { Boron } \\
41 \mathrm{x} \\
\text { PPL }\end{array}$ & $\begin{array}{r}49730 \\
11194 \\
21197 \\
2824 \\
3156\end{array}$ & $\begin{array}{l}0.40 \pm 0.02 \\
2.95 \pm 0.21 \\
0.56 \pm 0.17 \\
6.21 \pm 0.57 \\
6.97 \pm 0.58\end{array}$ \\
\hline $1426+015 \ldots \ldots \ldots \ldots$ & 1985 Jul 6 & $\begin{array}{l}\mathrm{ME} \\
\text { 3lx } \\
\mathrm{Al} / \mathrm{P} \\
\text { Boron }\end{array}$ & $\begin{array}{r}21810 \\
4689 \\
3599 \\
10056\end{array}$ & $\begin{array}{l}0.83 \pm 0.04 \\
4.63 \pm 0.39 \\
2.71 \pm 0.35 \\
0.27 \pm 0.12\end{array}$ \\
\hline $1501+106 \ldots \ldots \ldots \ldots$ & 1986 Feb 22 & $\begin{array}{l}\mathrm{ME} \\
3 \mathrm{~lx} \\
\mathrm{Al} / \mathrm{P}\end{array}$ & $\begin{array}{r}19860 \\
1998 \\
3713\end{array}$ & $\begin{array}{l}1.14 \pm 0.04 \\
9.55 \pm 0.86 \\
3.92 \pm 0.41\end{array}$ \\
\hline $2130+099 \ldots \ldots \ldots \ldots$ & 1985 Nov 14 & $\begin{array}{l}\text { ME } \\
\text { 31x } \\
\text { Al/P } \\
\text { Boron }\end{array}$ & $\begin{array}{r}26130 \\
4090 \\
3325 \\
15327\end{array}$ & $\begin{array}{l}0.49 \pm 0.03 \\
5.64 \pm 0.47 \\
3.26 \pm 0.39 \\
0.54 \pm 0.17\end{array}$ \\
\hline $2135-147 \ldots \ldots \ldots \ldots$ & 1984 May 12 & $\begin{array}{l}\mathrm{ME} \\
\text { 31x } \\
\mathrm{Al} / \mathrm{P} \\
\text { Boron }\end{array}$ & $\begin{array}{r}28560 \\
4863 \\
8033 \\
11691\end{array}$ & $\begin{array}{l}0.71 \pm 0.05 \\
2.14 \pm 0.27 \\
1.20 \pm 0.16 \\
0.34 \pm 0.12\end{array}$ \\
\hline
\end{tabular}

a Counts per second (units of $10^{-2}$ for the LE filters). 
TABLE 3

Single Power-Law Fits to the ME Data Assuming Galactic $N_{\mathrm{H}}$

\begin{tabular}{ccrccc}
\hline \hline Object & Channels $^{\mathrm{a}}$ & Flux $^{\mathrm{b}}$ & \multicolumn{1}{c}{$\alpha^{\mathrm{c}}$} & $N_{\mathrm{H}_{\mathrm{gal}}}{ }^{\mathrm{d}}$ & $\chi_{v}^{2} /$ d.o.f. \\
\hline $0003+199 \ldots \ldots$ & $7-35$ & 12.6 & $1.11_{-0.13}^{+0.13}$ & 4.00 & $1.01 / 27$ \\
$0007+106 \ldots \ldots$ & $7-35$ & 29.6 & $0.68_{-0.07}^{+0.08}$ & 6.09 & $0.75 / 27$ \\
$0026+129 \ldots \ldots$ & $7-25$ & 5.7 & $0.86_{-0.38}^{+0.39}$ & 4.93 & $0.67 / 17$ \\
$0049+171^{\mathrm{f}} \ldots \ldots$ & $7-25$ & 7.6 & $1.03_{-0.29}^{+0.29}$ & 4.26 & $1.98 / 17$ \\
$0052+251 \ldots \ldots$ & $7-26$ & 3.7 & $0.84_{-0.53}^{+0.53}$ & 4.50 & $1.28 / 18$ \\
$0637-752 \ldots \ldots$ & $7-29$ & 4.1 & $0.93_{-0.60}^{+0.64}$ & 4.80 & $0.61 / 21$ \\
$0804+761 \ldots \ldots$ & $7-25$ & 8.3 & $0.92_{-0.23}^{+0.23}$ & 3.12 & $0.93 / 17$ \\
$0923+129 \ldots \ldots$ & $7-24$ & 10.0 & $0.75_{-0.44}^{+0.44}$ & 4.03 & $0.98 / 16$ \\
$1211+143 \ldots \ldots$ & $7-25$ & 8.5 & $1.02_{-0.22}^{+0.23}$ & 2.83 & $1.38 / 17$ \\
$1217+023 \ldots \ldots$ & $7-25$ & 4.3 & $1.16_{-1.25}^{+1.25}$ & 1.97 & $1.00 / 17$ \\
$1219+756 \ldots \ldots$ & $7-28$ & 6.0 & $1.17_{-0.60}^{+0.67}$ & 2.74 & $0.84 / 20$ \\
$1226+023 \ldots \ldots$ & $7-35$ & 68.8 & $0.53_{-0.03}^{+0.03}$ & 1.80 & $1.00 / 27$ \\
$1229+204 \ldots \ldots$ & $7-29$ & 3.8 & $1.14_{-0.31}^{+0.33}$ & 2.58 & $1.07 / 21$ \\
$1426+015 \ldots \ldots$ & $7-35$ & 8.5 & $0.46_{-0.25}^{+0.25}$ & 2.64 & $0.81 / 27$ \\
$1501+106 \ldots \ldots$ & $7-35$ & 14.3 & $0.42_{-0.16}^{+0.16}$ & 2.23 & $1.53 / 27$ \\
$2130+099 \ldots \ldots$ & $7-30$ & 4.8 & $1.27_{-0.36}^{+0.38}$ & 4.20 & $0.84 / 22$ \\
$2135-147 \ldots \ldots$ & $7-30$ & 7.6 & $0.83_{-0.31}^{+0.33}$ & 4.45 & $0.99 / 22$ \\
\hline
\end{tabular}

a Channels used in the fit.

b Units of $10^{-12}$ ergs $\mathrm{cm}^{-2} \mathrm{~s}^{-1}$ in the energy range $2-8 \mathrm{keV}$.

c Power-law energy index with associated $90 \%$ confidence ranges for one interesting parameter $\left(\chi_{\min }^{2}+2.71\right.$; Avni 1976).

${ }^{\mathrm{d}}$ Units of $10^{20} \mathrm{~cm}^{-2}$, from Elvis, Lockman, \& Wilkes (1989), except for $0003+199$ (TP89), and 1226+023 (WE87).

${ }^{\mathrm{e}}$ Reduced $\chi^{2}$ and degrees of freedom.

f For PG $0049+171$ the Galactic $N_{\mathrm{H}}$ is excluded at more than $99 \%$ confidence by the two-parameter fit to the data. Leaving $N_{\mathrm{H}}$ free to vary, the best-fit parameters are $\alpha \simeq 3.5$ and $N_{\mathrm{H}} \sim 8 \times 10^{22} \mathrm{~cm}^{-2}$, with a reduced $\chi^{2}$ of 1.29 with 16 d.o.f. (see discussion in the text).

For several of the objects in our sample there are already published EXOSAT spectral data (Mrk 335, III Zw 2, 3C 273 [TP89], PG 0026+129 [Treves et al. 1988], and Mrk 841 [Arnaud et al. 1985]). In all cases but one, our fits are in good agreement, within the errors, with the published results. This is true both for the slopes of the hard power-law component and for the presence of soft excess emission, for which our $\alpha_{\text {steep }}$ values are consistent with those of TP89 for 3C 273 and Mrk 335. The only discrepancy is in the temperature of the fitted blackbody for Mrk 841 for which the value found by Arnaud et al. $(1985)(\sim 17 \mathrm{eV})$ is significantly lower than our best-fit value $(57 \mathrm{eV})$, but it must be noted that Arnaud et al. fitted a blackbody component combining data both in the ultraviolet and in the soft X-ray ranges. Additional comparisons with other nonEXOSAT results will be discussed in the next section.

\section{DISCUSSION}

\subsection{ME Results}

The hard X-ray emission specta of all the 17 AGNs in our sample can be well described by a single power law. The distribution of the ME spectral indices is shown in Figure 1, where the dashed part of the histogram represents objects with a $1 \sigma$ error on the slope smaller than 0.20 . In the same figure we show also the Gaussian which best fits the data in TP89. The distribution of spectral indices of our objects shows a wide range of slopes centered on $\alpha \sim 1.0$ and with a pronounced tail toward flat slopes. The average spectral index for our 17 objects is $\langle\alpha\rangle=0.89 \pm 0.06$, with an observed dispersion $\sigma=0.25$. Our distribution of observed ME spectral indices is significantly different (at $98 \%$ confidence level, according to the

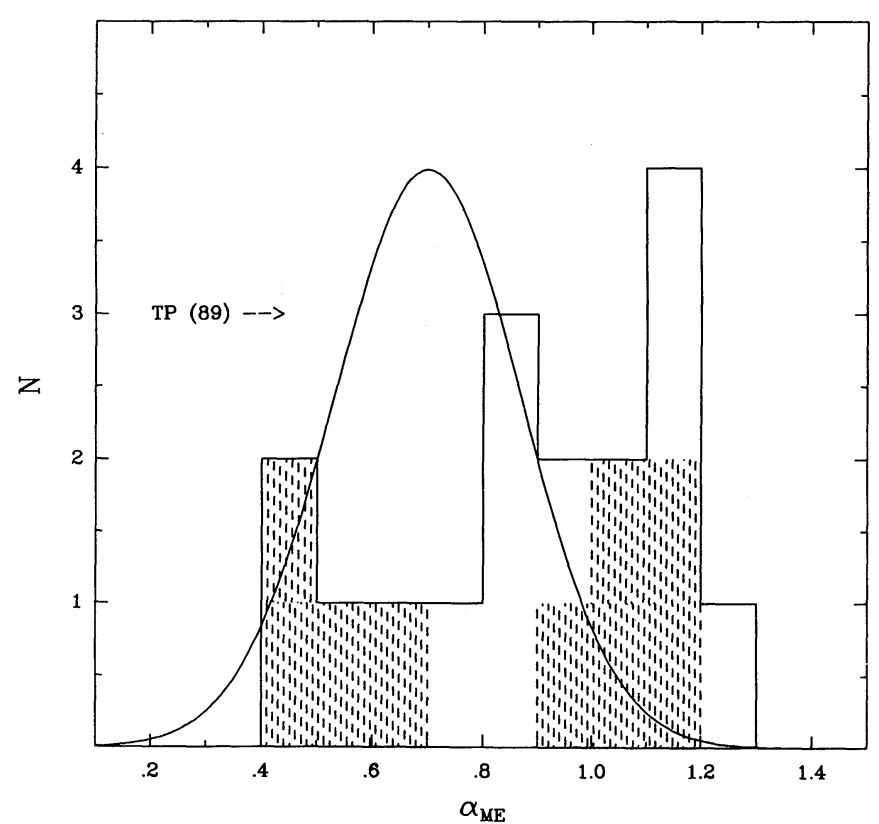

Fig. 1.-Distribution of the hard X-ray (2-10 keV) spectral energy indices of our sample. The dashed part of the histogram represents objects with $1 \sigma$ error on the slope smaller than 0.20 . The Gaussian curve is taken from Turner $\&$ Pounds (1989) and describes the best-fit energy index distribution of their sample.

Kolmogorov-Smirnov test) from that obtained with the same instrument by TP89 for hard X-ray-selected AGNs. In their sample the percentage of Seyfert 1 galaxies with $\alpha$ steeper than 1.0 is $7 \%$, to be compared with $41 \%$ in our sample. Two differences between these samples are immediately clear from inspection of Figure 2: first, our objects are on average more $\mathrm{X}$-ray luminous; second, in the range of overlapping lumi-

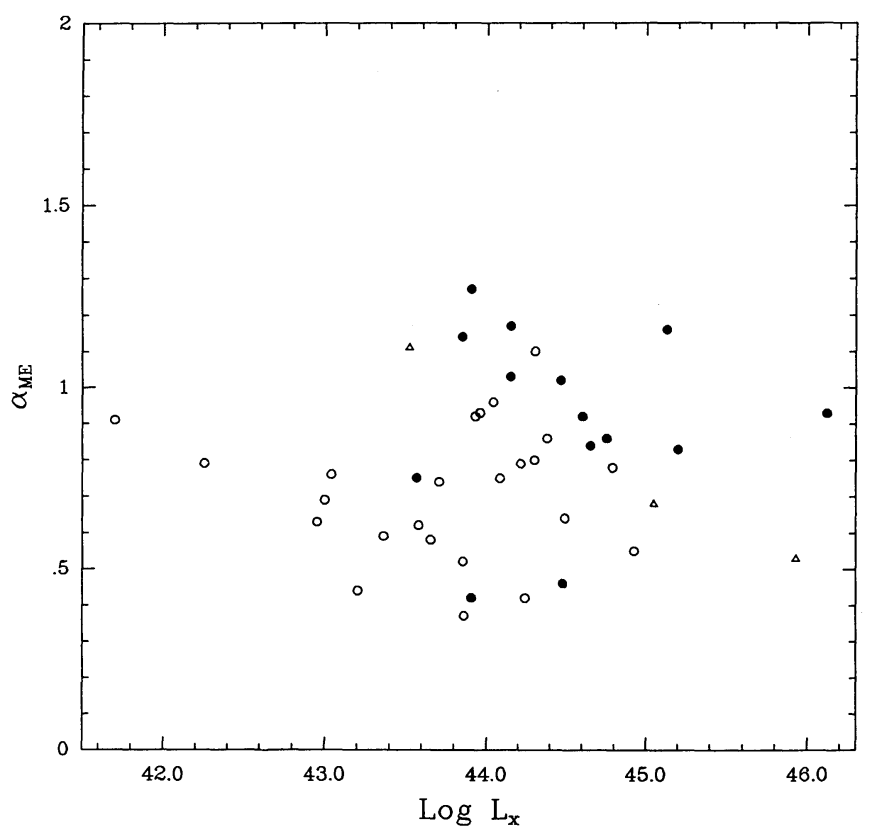

FIG. 2.-ME spectral energy index values vs. the logarithm of the X-ray luminosity (ergs $\mathrm{s}^{-1}$ ). We have marked with filled circles the objects of our sample, with open circles the Seyfert 1 galaxies and quasars in the Turner $\&$ Pounds (1989) sample and with open triangles the three objects in common. 
TABLE 4

Single PoWER-LAW Fits to THE ME + LE Data

\begin{tabular}{|c|c|c|c|c|c|c|}
\hline $\begin{array}{l}\text { Object } \\
\text { (1) }\end{array}$ & $\begin{array}{c}\alpha \\
(2)\end{array}$ & $\frac{N_{\mathrm{H}^{\mathrm{a}}}}{N_{\mathrm{H}_{\mathrm{ga}}{ }^{\mathrm{a}}}}$ & $\begin{array}{c}\chi_{v}^{2} / \text { d.o.f. }^{b} \\
\text { (4) }\end{array}$ & $\begin{array}{l}\Delta \chi^{2} / \text { n.o.f. }^{c} \\
\text { (5) }\end{array}$ & $\begin{array}{c}\text { Classification } \\
\text { (6) }\end{array}$ & $\begin{array}{l}R_{\text {ex }}^{\mathrm{d}} \\
(7)\end{array}$ \\
\hline \multirow[t]{2}{*}{$0003+199 \ldots \ldots \ldots$} & $0.68_{-0.03}^{+0.03}$ & $0.0-0.007$ & $2.30 / 29$ & $39.75 / 3$ & Soft excess & $2.74_{-0.38}^{+0.48}$ \\
\hline & $1.64_{-0.05}^{+0.05}$ & 4.00 & $6.47 / 30$ & $167.05 / 3$ & & \\
\hline \multirow[t]{2}{*}{$0007+106 \ldots \ldots \ldots$} & $0.68_{-0.10}^{+0.10}$ & $6.0_{-1.8}^{+2.4}$ & $0.72 / 28$ & $4.86 / 2$ & Single power law & $0.00_{-0.09}^{+0.09}$ \\
\hline & $0.68_{-0.04}^{+0.04}$ & 6.09 & $0.70 / 29$ & $0.08 / 2$ & & \\
\hline \multirow[t]{2}{*}{$0026+129 \ldots \ldots \ldots$} & $1.00_{-0.38}^{+0.44}$ & $4.0_{-2.8}^{+7.3}$ & $0.65 / 19$ & $1.04 / 3$ & Single power law & $0.49_{-0.39}^{+0.57}$ \\
\hline & $1.07_{-0.11}^{+0.10}$ & 4.93 & $0.63 / 20$ & $1.21 / 3$ & & \\
\hline \multirow{2}{*}{$0049+171 \ldots \ldots \ldots$} & $1.02_{-0.40}^{+0.44}$ & $8.1_{-5.5}^{+10.9}$ & $1.91 / 18$ & $13.66 / 2$ & Single power law & $-0.39_{-0.18}^{+0.23}$ \\
\hline & $0.79_{-0.08}^{+0.07}$ & 4.26 & $1.90 / 19$ & $2.45 / 2$ & & \\
\hline \multirow[t]{2}{*}{$0052+251 \ldots \ldots \ldots$} & $0.95_{-0.42}^{+0.52}$ & $2.1_{-2.1}^{+5.3}$ & $1.22 / 19$ & $3.65 / 2$ & Single power law & $1.14_{-0.77}^{+1.40}$ \\
\hline & $1.23_{-0.12}^{+0.12}$ & 4.50 & $1.25 / 20$ & $2.01 / 2$ & & \\
\hline \multirow[t]{2}{*}{$0637-752 \ldots \ldots \ldots$} & $0.95_{-0.82}^{+0.96}$ & $11.9_{-11.2}^{+32.5}$ & $0.61 / 21$ & $0.04 / 1$ & Single power law & $-0.45_{-0.28}^{+0.60}$ \\
\hline & $0.61_{-0.17}^{+0.16}$ & 4.80 & $0.62 / 22$ & $0.80 / 1$ & & \\
\hline \multirow[t]{2}{*}{$0804+761 \ldots \ldots \ldots$} & $1.02_{-0.22}^{+0.22}$ & $2.7_{-1.4}^{+2.2}$ & $1.18 / 19$ & $6.72 / 3$ & Single power law & $0.42_{-0.27}^{+0.34}$ \\
\hline & $1.07_{-0.08}^{+0.06}$ & 3.12 & $1.14 / 20$ & $6.99 / 3$ & & \\
\hline \multirow[t]{2}{*}{$0923+129 \ldots \ldots \ldots$} & $0.87_{-0.31}^{+0.36}$ & $2.9_{-1.9}^{+5.0}$ & $0.94 / 18$ & $1.12 / 3$ & Single power law & $0.51_{-0.44}^{+0.71}$ \\
\hline & $0.97_{-0.11}^{+0.10}$ & 4.03 & $0.91 / 19$ & $1.57 / 3$ & & \\
\hline \multirow[t]{2}{*}{$1211+143 \ldots \ldots \ldots$} & $1.48_{-0.06}^{+0.12}$ & $1.4_{-0.3}^{+0.6}$ & $2.63 / 19$ & $28.44 / 3$ & Soft excess & $3.87_{-0.83}^{+1.10}$ \\
\hline & $1.76_{-0.06}^{+0.06}$ & 2.83 & $3.12 / 20$ & $38.99 / 3$ & & \\
\hline \multirow[t]{2}{*}{$1217+023 \ldots \ldots \ldots$} & $0.94_{-0.38}^{+0.51}$ & $1.5_{-1.5}^{+4.1}$ & $0.96 / 18$ & $0.12 / 2$ & Single power law & $-0.27_{-0.40}^{+1.96}$ \\
\hline & $1.02_{-0.14}^{+0.19}$ & 1.97 & $0.92 / 19$ & $0.32 / 2$ & & \\
\hline \multirow[t]{2}{*}{$1219+756 \ldots \ldots \ldots$} & $0.89_{-0.36}^{+0.75}$ & $1.7_{-1.7}^{+11.7}$ & $0.83 / 21$ & $0.72 / 2$ & Single power law & $-0.28_{-0.29}^{+0.62}$ \\
\hline & $1.02_{-0.13}^{+0.12}$ & 2.74 & $0.81 / 22$ & $1.10 / 2$ & & \\
\hline \multirow[t]{2}{*}{$1226+023 \ldots \ldots \ldots$} & $0.53_{-0.05}^{+0.05}$ & $0.6_{-0.2}^{+0.2}$ & $2.63 / 29$ & $49.59 / 3$ & Soft excess & $1.00_{-0.09}^{+0.11}$ \\
\hline & $0.66_{-0.04}^{+0.03}$ & 1.80 & $5.79 / 30$ & $146.50 / 3$ & & \\
\hline \multirow[t]{2}{*}{$1229+204 \ldots \ldots \ldots$} & $1.39_{-0.18}^{+0.19}$ & $1.3_{-0.6}^{+0.8}$ & $1.05 / 24$ & $2.64 / 4$ & Soft excess & $1.88_{-0.59}^{+0.91}$ \\
\hline & $1.64_{-0.05}^{+0.06}$ & 2.58 & $1.38 / 25$ & $11.97 / 4$ & & \\
\hline \multirow[t]{2}{*}{$1426+015 \ldots \ldots \ldots$} & $0.38_{-0.06}^{+0.06}$ & $0.0-0.023$ & $0.90 / 29$ & $4.41 / 3$ & Soft excess & $3.26_{-0.88}^{+1.17}$ \\
\hline & $1.10_{-0.07}^{+0.07}$ & 2.64 & $1.65 / 30$ & $27.54 / 3$ & & \\
\hline \multirow[t]{2}{*}{$1501+106 \ldots \ldots \ldots$} & $0.46_{-0.06}^{+0.10}$ & $0.0-0.051$ & $1.49 / 28$ & $0.41 / 2$ & Soft excess & $3.94_{-0.70}^{+0.84}$ \\
\hline & $1.10_{-0.09}^{+0.07}$ & 2.23 & $3.34 / 29$ & $55.53 / 2$ & & \\
\hline \multirow[t]{2}{*}{$2130+099 \ldots \ldots \ldots$} & $1.45_{-0.30}^{+0.32}$ & $2.6_{-1.4}^{+2.5}$ & $0.85 / 24$ & $1.97 / 3$ & Single power law & $1.19_{-0.59}^{+0.92}$ \\
\hline & $1.66_{-0.08}^{+0.08}$ & 4.20 & $0.90 / 25$ & $3.93 / 3$ & & \\
\hline \multirow[t]{2}{*}{$2135-147 \ldots \ldots \ldots$} & $0.78_{-0.28}^{+0.34}$ & $2.4_{-1.7}^{+4.3}$ & $0.92 / 24$ & $0.11 / 3$ & Single power law & $0.32_{-0.31}^{+0.43}$ \\
\hline & $0.96_{-0.10}^{+0.09}$ & 4.45 & $0.95 / 25$ & $1.81 / 3$ & & \\
\hline
\end{tabular}

${ }^{a}$ Units of $10^{20} \mathrm{~cm}^{-2}$

${ }^{b}$ Reduced $\chi^{2}$ and degrees of freedom.

c Increment in the $\chi^{2}$ value resulting from the addition to the ME data of the data for the LE filters over the number of LE filters (n.o.f.).

d Ratio of the observed minus predicted counts to the predicted counts in the Al/P and 3000 Lexan filters. The predicted counts are computed by extrapolating the ME best fit. The errors are $1 \sigma$.

nosities our objects tend to have a steeper spectral index (although a few flat spectrum objects are also present in our sample). Part of the latter difference can be explained by the different selection criteria of the two samples, as Elvis et al. (1986) concluded (see also Zamorani 1984). While ours is a flux-limited sample selected in the soft X-ray domain, the TP89 sample comprises objects which have been primarily selected in the hard X-ray domain. Each of the two selections has its own biases, ours against flat spectra, theirs against steep spectra.

However, essentially the same difference is present between our data and the Einstein MPC data of 20 Seyfert 1 galaxies of Urry et al. (1989), who claim that their sample, like ours, is soft $\mathrm{X}$-ray flux-limited. This is probably not completely true, because the original IPC Seyfert sample from which this subsample has been derived (Kruper et al. 1990) contains four objects (i.e., about $20 \%$ of the Urry et al. 1989 sample) with an IPC flux greater than their stated threshold, for which, however, no MPC spectra are presented. It is likely that the spectra of these objects are, on average, steeper than the others, thus explaining the lack of useful MPC data. Assuming that the spectral slope of these four objects are of the order of the steepest object of the sample, the mean of their entire distribution would change from 0.70 to 0.79 , thus becoming almost consistent with ours.

A variety of models and emission mechanisms have been proposed to explain the X-ray spectral properties of AGNs, and in particular the "universal" hard spectral index of 0.7 (e.g., Mushotzky 1984). Our results, which show that the distribution of the observed spectral indices is a function of the selection criteria of the samples, lead us to suggest that the true distribution of hard X-ray (above $\sim 2 \mathrm{keV}$ ) spectral indices is 
TABLE 5

Double Power-Law and Blackbody + Power-Law Fits

\begin{tabular}{|c|c|c|c|c|c|c|c|c|c|}
\hline $\begin{array}{c}\text { OBJECT } \\
\text { (1) }\end{array}$ & $\alpha_{\mathrm{ME}}^{\mathrm{a}}$ & $\begin{array}{c}\alpha_{\text {steep }} b \\
(3)\end{array}$ & $\begin{array}{c}\chi_{v}^{2} / \text { d.o.f. } \\
\text { (4) }\end{array}$ & $\begin{array}{c}\mathrm{P}\left(\chi^{2}\right)^{\mathrm{d}} \\
(5)\end{array}$ & $\begin{array}{c}E_{\text {break }}{ }^{\mathrm{e}} \\
\text { (6) }\end{array}$ & $\begin{array}{c}k T^{\mathrm{f}} \\
(7)\end{array}$ & $\begin{array}{c}\chi_{v}^{2} / \text { d.o.f. } \\
(8)\end{array}$ & $\begin{array}{l}P\left(\chi^{2}\right)^{d} \\
(9)\end{array}$ & $\begin{array}{c}E_{\text {break }}{ }^{\mathrm{e}} \\
(10)\end{array}$ \\
\hline $0003+199 \ldots \ldots \ldots$ & $1.11_{-0.13}^{+0.13}$ & $4.22_{-0.49}^{+0.58}$ & $1.62 / 30$ & 0.018 & 0.48 & $46_{-7}^{+10}$ & $1.01 / 30$ & 0.45 & 0.49 \\
\hline $1211+143 \ldots \ldots \ldots$ & $1.02_{-0.22}^{+0.23}$ & $3.33_{-0.42}^{+0.66}$ & $1.45 / 20$ & 0.085 & 0.67 & $57_{-14}^{+19}$ & $1.18 / 20$ & 0.26 & 0.61 \\
\hline $1426+015 \ldots \ldots \ldots$ & $0.46_{-0.25}^{+0.25}$ & $2.66_{-0.49}^{+1.09}$ & $0.91 / 30$ & 0.610 & 0.66 & $79_{-27}^{+32}$ & $0.77 / 30$ & 0.81 & 0.76 \\
\hline $1501+106 \ldots \ldots \ldots$ & $0.42_{-0.16}^{+0.16}$ & $2.87_{-0.48}^{+1.07}$ & $1.45 / 29$ & 0.057 & 0.64 & $57_{-19}^{+29}$ & $1.43 / 29$ & 0.064 & 0.54 \\
\hline
\end{tabular}

Medium-energy power-law energy index (from the ME best fit), with associated $90 \%$ confidence ranges.

b Low-energy power-law energy index, with associated $90 \%$ confidence ranges.

c Reduced $\chi^{2}$ and degrees of freedom.

d Probability of exceeding by chance the observed value of $\chi^{2}$.

e Break energy in keV (at the source), defined as the intersection between the soft and hard component.

f Blackbody temperature in $\mathrm{eV}$, with associated $90 \%$ confidence ranges.

wider than previously suggested and probably shifted toward steeper spectra.

Ginga observations have revealed several new features in the hard X-ray spectra of low-luminosity AGNs, in particular a flattening of the spectrum for energies $\gtrsim 10 \mathrm{keV}$ ("hump"), which has been interpreted either as due to partial coverage of an underlying power law continuum by thick cold gas (Piro, Matsuoka, \& Yamauchi 1989; Matsuoka et al. 1990) or, most likely, as the signature of reprocessed X-rays by relatively cold $\left(<10^{6} \mathrm{~K}\right)$ gas in the proximity of the central source (Pounds et al. 1990). In the last model the observed "universal" slope $\alpha \simeq 0.7$ would result from an injected spectrum with slope $\alpha \sim 0.9$. On the other hand, a recent analysis of Ginga spectral data $(2-20 \mathrm{keV})$ for a sample of high-luminosity AGNs finds a mean spectral index $\langle\alpha\rangle=0.81$ and no indication of a substantial reflection component as in the low-luminosity AGNs (Williams et al. 1991). In this framework our result, $\left\langle\alpha_{\mathrm{ME}}\right\rangle \sim$ 0.9 , can be interpreted in two different ways: either the reflected component is almost absent in the objects of our sample and we see mainly the direct component, or, if the reflection model holds also for these objects (but this has still to be demonstrated) their intrinsic emission spectrum has to be even steeper.

\section{2. $M E+L E$ Results}

The addition of the low energy (LE) data has allowed the analysis of the X-ray spectra over an energy interval spanning about two orders of magnitude $(0.1-8 \mathrm{keV})$. We find that for about $2 / 3$ of the sample (11/17), a single power-law fit is consistent with the data over the entire energy range. The remaining six objects show a clear indication of soft excess emission in the low-energy filters. This percentage of objects with significant soft excess is consistent with that $(31 \%)$ found by TP89 for Seyfert 1 galaxies. The X-ray spectra of a sample of 14 quasars have been recently measured by Masnou et al. (1991), over approximately the same energy range, with the IPC and MPC instruments of the Einstein Observatory. They find a higher percentage $(57 \%)$ of objects with soft excess emission at low energy (below $0.5 \mathrm{keV}$ ). Four of the six objects for which we detect soft excess emission are in common with this sample; for three out of these four objects, the soft excess emission has been also recovered by Masnou et al. (1991). Vice versa, they find soft excess in two of five of the remaining objects in common for which a single power law gives a good fit to our data.
We note that the objects with soft excess in our sample tend to lie in regions of lower Galactic $N_{\mathrm{H}}$ than those which are consistent with a single power law. For example, soft excess is detected in five out of eight objects with a Galactic $N_{\mathbf{H}}$ lower than $3.5 \times 10^{20}$, and in one out of nine objects with Galactic $N_{\text {H }}$ greater than this limit. The same effect is present at some level also in the TP89 data: the percentage of Seyfert 1 galaxies with soft excess in their sample increases to $55 \%$ when the objects with Galactic $N_{\mathrm{H}}<3.5 \times 10^{20}$ are considered. Some correlation between detection of soft excess and Galactic $N_{\mathrm{H}}$ is qualitatively expected because the same amount of soft excess is more easily detected in the absence of any kind of intervening absorption. To obtain a more quantitative estimate of the possible importance of this effect we have performed the following test: first, we have created simulated data for the six objects with soft excess emission (Table 5) assuming that the true spectrum of each source is the one found from the best fit with a double power law, or with the blackbody plus a power law, models. For each source we have assumed the actually observed background. Then we have applied our fitting procedure on these simulated data through the actually used filters searching for the minimum amount of Galactic $N_{\mathrm{H}}$ above which there would be no statistically significant difference between the single and the double power law (or blackbody plus power law) fits: had these objects been in a region of Galactic $N_{\mathbf{H}}$ greater than this minimum value, we would have classified their spectra as consistent with a single power law. For five objects we find that this minimum value of $N_{\mathrm{H}}$ lies between $8 \times 10^{20}$ and $1.4 \times 10^{21} \mathrm{~cm}^{-2}$. For the remaining object $(\mathrm{PG} 1229+204)$ the minimum value of $N_{\mathrm{H}}$ is about $4 \times 10^{20}$. Since these values (with the exception of $P G$ $1229+204)$ are much higher than the Galactic $N_{\mathrm{H}}$ of all the objects in our sample, we can conclude that a strong soft excess, if present, would have been detected in the objects classified as single power law, all of which have a Galactic $N_{H}$ lower than $6 \times 10^{20} \mathrm{~cm}^{-2}$. A more marginal excess (e.g., PG $1229+204)$, however, could have escaped detection in objects with Galactic $N_{\mathrm{H}}>4 \times 10^{20}$. As a consequence, the fact that a large fraction of soft excess objects in our sample is located in regions of low Galactic $N_{\mathrm{H}}$ appears to be accidental (see also $\S 3.3)$.

To give a more quantitative estimate of the number of objects for which the detection of a weak excess could have been missed by our fitting procedure, we have computed for 
each object the quantity $R_{\text {ex }}$ (last column of Table 4) defined as the ratio of the observed minus predicted counts in the low energy filters to the predicted counts computed by extrapolating to low energy the ME best fit. For the six objects classified "soft excess," the value of $R_{\mathrm{ex}}$ is significantly larger than zero, thus confirming the previous classification. For five of 11 objects for which a single power law gives an acceptable fit over the entire energy range, we find a deficit of counts $\left(R_{\mathrm{ex}}<\right.$ $0)$, while for the remaining six we find some excess $\left(R_{\mathrm{ex}}>0\right)$ with a significance between $1 \sigma$ and $2 \sigma$. The data of the latter objects have been fitted with a double power-law model with the high-energy parameters fixed from the ME fit. To be consistent with the results shown in Table 5 , we varied $\alpha_{\text {steep }}$ in the range 2.5-4.0 leaving as a free parameter the low-energy normalization or, equivalently, the break energy. The results show that the break energies above which the fits become significantly worse than the single power-law fits lie between 0.2 and $0.5 \mathrm{keV}$ depending on the adopted steepness of the low-energy power law and on the value of $R_{\text {ex }}$. These values have to be compared with the break energies derived for the objects with soft excess emission, which are in the energy range $0.35-0.75$ $\mathrm{keV}$. The partial overlap between the energy range of the break energies for objects with and without detected soft excess suggests that we cannot exclude that also some of the AGNs in our sample whose spectra are consistent with a single power law do really have some kind of soft excess, although confined to softer X-ray energies.

Also it should be noted that the objects with detected soft excess emission tend to have better constrained ME spectra, i.e., smaller error on $\alpha$. For this reason they have, on average, smaller errors on the expected extrapolated counts at soft energy so that the soft excess, if present can be more easily detected. On the basis of the results of the two previous tests and of this consideration, we conclude that the observed percentage $(35 \%)$ of soft excess objects in our sample should be considered as a lower limit on the true frequency of such objects. Even more so if we consider that the presence of a possible absorption intrinsic to the objects, which was assumed to be absent in our fitting procedure, would cause a systematic underestimate of the soft excess strength. We have quantified the effect of additional absorbing material assuming an intrinsic column density at the source of $2 \times 10^{20} \mathrm{~cm}^{-2}$. The corresponding increase of the X-ray opacity results in the detection of soft excess in one more quasar, i.e., PG $2130+099$.

An independent indication of the possible presence of some excess emission at low energies in most of the objects is seen in Figure 3, which shows the distribution of $\Delta N_{\mathrm{H}}$, defined as the difference between the best $N_{\mathrm{H}}$ value obtained through the fit of a single power law to the ME and LE data and the Galactic one. If all the objects had a negligible intrinsic absorbing column, this distribution would be symmetric about zero with a spread consistent with the expected small-scale fluctuations of $N_{H}$ in our Galaxy and the measurement errors. Even excluding the six objects with detected soft excess (marked with an $\mathrm{S}$ in the histogram), the distribution is significantly displaced toward negative values of $\Delta N_{\mathrm{H}}$ : only two objects have $N_{\mathrm{H}}$ $(E X O S A T)>N_{\mathrm{H}}(21 \mathrm{~cm})$. Following WE87, this can be taken as an evidence of a general presence of some soft excess in the population, although not detectable in the analysis of the spectra of the single objects. Moreover, this is consistent with the average steepening of the fits to the ME + LE data with respect to the ME ones: for the 11 objects fitted with a single power law, we find an average $\mathrm{LE}+\mathrm{ME}$ spectral index

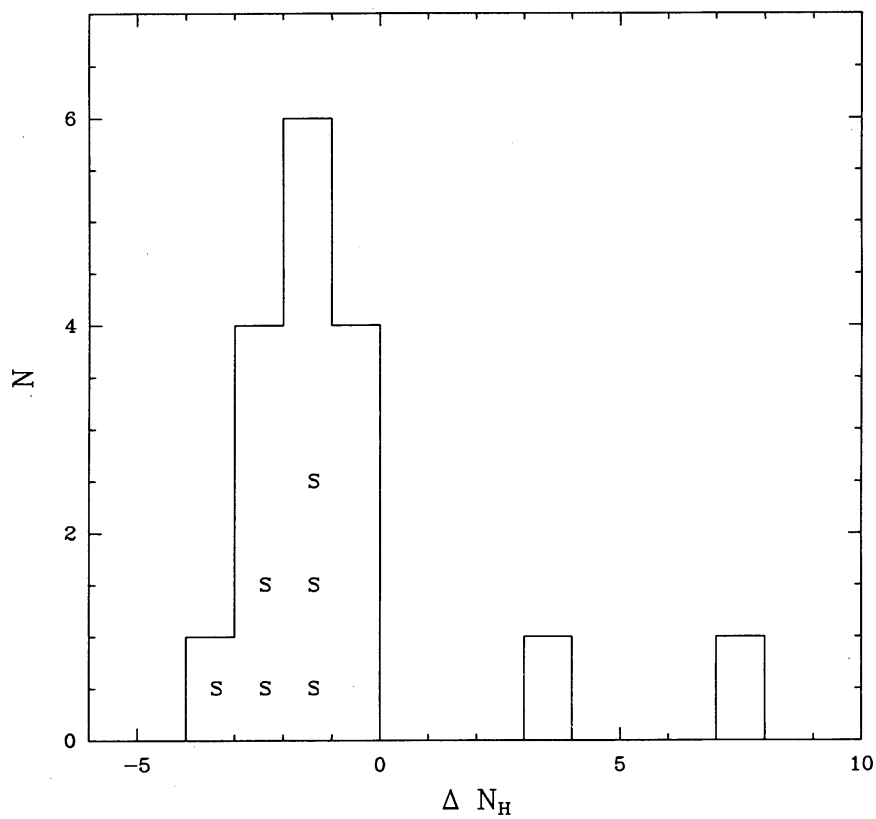

FIG. 3.-Distribution of $\Delta N_{\mathrm{H}}$ (in units of $10^{20} \mathrm{~cm}^{-2}$ ) defined as the difference between the $N_{\mathrm{H}}$ value obtained through the fit of a single power law over the full energy range $(\sim 0.1-10 \mathrm{keV})$ and the Galactic value obtained by radio measurements (Elvis, Lockman, \& Wilkes 1989). The objects with detected soft excess emission are marked with an $\mathrm{S}$.

$\langle\alpha\rangle=1.01 \pm 0.08$, while for the same objects the mean of the ME spectral indices are $0.95 \pm 0.05$.

For the objects with a significant soft excess the resulting parameters from the fits with two power laws or a blackbody plus a power law are qualitatively similar to each other. Because of the lack of detailed energy resolution of the LE detectors the spectral shape of the soft excess is not well constrained, even assuming a fixed underlying power law with the ME best-fit parameters, and for most of these objects we cannot discriminate between the power-law and the blackbody models. However, it is interesting to note that for five out of six objects the blackbody model gives a better fit than the powerlaw model and the difference between the two models is statistically significant (see cols. [5] and [9] of Table 5) for 3C 273 and Mrk 335, i.e., the two sources with the highest soft flux. For these objects the difference remains significant even considering the uncertainty in the underlying power-law slope. In particular, for each of the objects we find that (1) for the two power-law model, the best-fit slope at low energy falls in the range 2.7-4.2, much steeper than the high-energy slope; and (2) for the blackbody plus power-law model, the best-fit blackbody temperatures are in the range of $40-80 \mathrm{eV}$, with associated errors of the order of $30 \%$. This range of temperatures is at variance with the bimodal distribution $(k T<10 \mathrm{eV}$ or $k T \simeq 200-300 \mathrm{eV}$ ) suggested on the basis of an analysis of IPC data of Seyfert 1 galaxies by Urry et al. (1989), who, however, note that their result is affected by the discontinuous response of the IPC in the energy range $250-600 \mathrm{eV}$. (3) For both fitting models, the nominal energy of intersection of the soft and hard component is in the range $0.35-0.75 \mathrm{keV}$.

\subsection{X-Ray Spectra versus Radio-Loudness}

We have searched for correlations between the hard X-ray spectral index and other physical parameters of the sources, but none has been found. Specifically, no significant corre- 

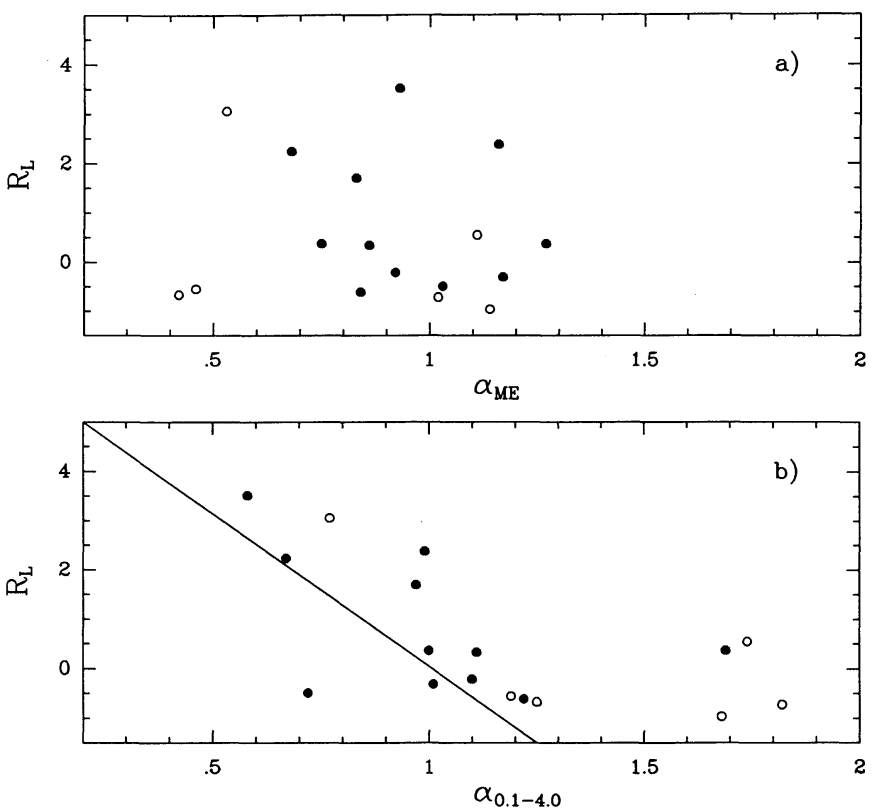

FIG. 4.-(a) ME spectral energy index values vs. the radio loudness index $R_{L}$. (b) $0.1-4.0 \mathrm{keV}$ energy index values vs. the radio-loudness index $R_{L}$. We have marked with open circles the objects with soft excess emission and with filled circles, the others. It must be noted that most of our data points lie above the line representing the WE87 correlation.

lation has been found with the intrinsic optical luminosity nor with the redshift spanning from 0.025 to 0.25 (with one object at $z=0.65$ ) nor with the X-ray luminosity of the objects, which encompasses about three decades. As discussed in $\S 3.1$, the fact that the mean spectral slope of our sample, containing mostly high-luminosity objects, is steeper than the one found by TP89 for lower luminosity AGNs, is probably due to the different selection effects (i.e., soft versus hard X-ray-selected objects).

In agreement with the findings of TP89 no obvious correlation is evident also between the ME spectral indices $\left(\alpha_{\mathrm{ME}}\right)$ and the strength of the radio emission $\left(R_{L}\right)$, defined as $R_{L}=$ $\log \left(f_{R} / f_{B}\right)$, where $f_{R}$ and $f_{B}$ are the flux densities at $5 \mathrm{GHz}$ and $2500 \AA$ (Fig. $4 a$ ). Such a correlation was found by WE87 in the IPC band with the radio-loud objects grouped around $\alpha \simeq 0.5$ and the radio-quiet ones around $\alpha \simeq 1.0$. Following WE87 we define a quasar to be radio-loud when $R_{L}>1$. In order to understand the reason for this apparent discrepancy (note that 11 of 17 objects in our sample are in common with the WE87 sample), we have performed spectral fits to our data in the $0.1-4.0 \mathrm{keV}$ energy range (similar to the IPC range, $0.2-3.5$ $\mathrm{keV}$ ), with $N_{\mathrm{H}}=N_{\mathrm{H}_{\mathrm{gal}}}$. The results (Fig. $4 b$ ) show that in this softer energy range there is a clear trend with radio-quiet quasars having steeper EXOSAT slopes than radio-loud quasars, in agreement with the WE87 work. However, for the 11 objects in common with WE87 we find that for the same $N_{\mathrm{H}}$ values, our slopes are systematically steeper, with an average $\Delta \alpha=\alpha_{\text {ours }}-\alpha_{\text {WE87 }}$ of the order of $0.2-0.3$. This difference could be due to either a systematic effect between the two detectors (IPC and LE/ME), or the slightly softer energy interval considered for our spectral fit results. It is interesting to note that the mean slope for the radio-quiet objects in the $0.1-4.0 \mathrm{keV}$ energy range $(\langle\alpha\rangle \simeq 1.2-1.3)$ agrees with the mean slope (1-2 keV) of the sources, mostly AGNs, in the ROSAT Medium Sensitivity Survey (Hasinger, Schmidt, \& Truemper 1991). Here we point out the existence of four objects with extremely EXOSAT steep slopes $(\alpha>1.6)$ in the $0.1-4.0 \mathrm{keV}$ energy range (PG 0003+199, PG $1211+143$, PG $1229+204$, and PG 2130+099). One of them (PG 1211+143) was the only object with a very steep slope $(\alpha=1.8)$ also in the IPC energy range and was classified as exceptional by WE87. Our results indicate that these steep slopes are probably quite common in quasar spectra.

The other apparently significant correlation in our data is between the presence of soft excess and radio-loudness. Five out of six objects with detected soft excess emission are radioquiet, and four of these are among the last five most radioquiet objects in the sample (see col. [9] in Table 1). The only radio-loud object with soft excess $(3 \mathrm{C} 273)$ is somewhat peculiar with respect to the other objects in the same class in that its value of $R_{\mathrm{ex}}$ is significantly lower than the mean value of the other five objects $\left(R_{\mathrm{ex}} \sim 3\right)$, so that the detection of the soft excess in 3C 273 is due mainly to its high X-ray flux and signal-to-noise ratio spectrum, about one order of magnitude larger than the average flux of the other objects in the sample.

In the previous section we have discussed the apparent correlation between the soft excess and the amount of Galactic $N_{\mathrm{H}}$. Therefore the soft excess (or equivalently, the excess counts $R_{\text {ex }}$ ) appears to be correlated with both $N_{\mathrm{H}}$ and $R_{L}$. In order to determine which of the two correlations is the fundamental one, we have computed the Kendall partial rank-order correlation coefficient $T_{x y, z}$ (Siegel \& Castellan 1988), which allows one to compute the probabilities of correlation for each pair of variables with the other one held constant. The only significant (anti-)correlation resulting from this test is that between the excess of counts, $R_{\mathrm{ex}}$, and the radio-loudness, $R_{L}$. This result is consistent with the conclusion reached in $\S 3.2$, that the apparent correlation between soft excess and $N_{\mathrm{H}}$ is mostly accidental.

The different spectral behavior of the radio-loud and radioquiet quasars is also shown in Figure 5 where we plot the ME spectral slopes versus the $0.1-4.0 \mathrm{keV}$ slopes. Two facts are

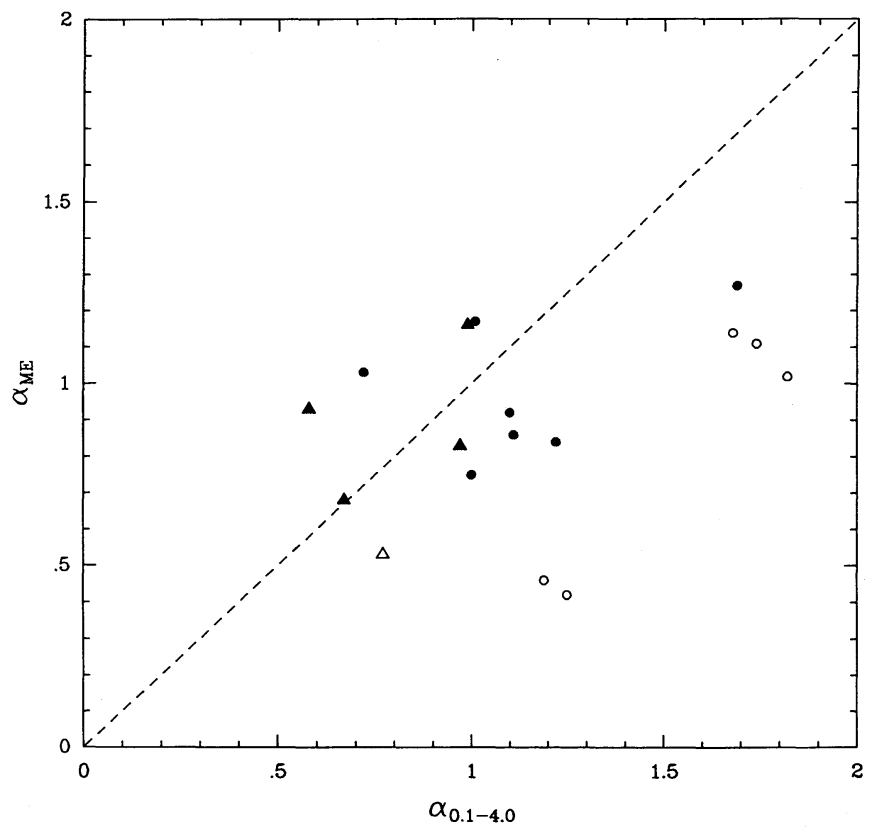

Fig. 5. - ME spectral energy index values vs. the $0.1-4.0 \mathrm{keV}$ ones. Circles and triangles represent radio-quiet and radio-loud objects, respectively. The objects with soft excess are represented with open symbols. 
immediately clear from the figure: first, the radio-loud objects (triangles) are spread around the line of equal spectral indices and do not show any change of slope over the entire energy range; second, the radio-quiet objects (circles) tend to have systematically steeper slopes in the $0.1-4.0 \mathrm{keV}$ energy range. This is true also for those objects without soft excess.

The observed anticorrelation between $R_{L}, R_{\mathrm{ex}}$ and the data shown in Figure 5 can be interpreted in two physically different ways by adopting simple two-component models. Either the radio-quiet objects may have an enhanced soft emission on the top of the hard power-law component, which in this case would be the same in both radio-quiet and radio-loud quasars, or, alternatively, the radio-loud ones may have a relatively stronger power-law component with a constant slope from low to medium energy. The existence of this enhanced power-law component would thus make more difficult the detection of the soft excess in their spectra and would be consistent with the fact that the radio-loud quasars are stronger X-ray emitters (about a factor 3) than the radio-quiet ones (Zamorani et al. 1981). Both these alternative pictures are perfectly consistent with our EXOSAT data. However, if the explanation of the systematic difference between our $0.1-4.0 \mathrm{keV}$ spectral slopes and the IPC slopes for the objects in our sample is not due to instrumental calibration differences between EXOSAT and the Einstein IPC, but rather only to the slightly softer spectral extension of EXOSAT, then the emission spectra of the sources may be much more complex and the simple modeling described above needs to be modified.

\section{CONCLUSIONS}

We have analyzed simultaneous LE and ME EXOSAT data to determine the X-ray spectra of 17 objects selected by their soft X-ray flux (mostly PG quasars and Seyfert 1 nuclei). The most important results of our spectral survey can be summarized as follows:

1. The hard X-ray emission spectra (above $\sim 2 \mathrm{keV}$ ) of all the AGNs in our sample can be described by a single powerlaw model. These measured spectral indices show a wide variety of power-law slopes with $\alpha$ spanning from 0.4 to 1.3 . The average slope is $\langle\alpha\rangle=0.89 \pm 0.06$, but the distribution is centered on $\alpha=1.0$. The narrow observed range of slopes around $\alpha \simeq 0.7$ in high-energy-selected samples is a result of their tendency to select the most " $2-10 \mathrm{keV}$ bright" AGNs, which tend to be those with the flattest X-ray spectra over the whole $2-10 \mathrm{keV}$ range. These results suggest that the true distribution of hard X-ray spectral indices is wider and probably steeper than previously suggested.

2. Approximately one-third (six out of 17) of the sources in our sample show evidence for soft excess emission in the lowenergy filters (below $1 \mathrm{keV}$ ). For these objects, a power law plus a soft excess modeled by steep power law or by the high-energy tail of a blackbody is a significantly better description of the data than a single power law over the energy range $0.1-10 \mathrm{keV}$. For the objects with the highest signal-to-noise ratio data, the blackbody model seems to be preferred to the two power-law model.

3. The best-fit slopes of the steep power-law fits at low energies are in the range $\alpha=2.7-4.2$, while the preferred temperatures of the fitted blackbodies are in the range $40-80 \mathrm{eV}$. The "break" energy between the soft and hard component is in the range $0.35-0.75 \mathrm{keV}$ at the source.

4. There is no obvious dependence of ME energy index with the other physical parameters of the sources such as the optical and X-ray luminosity, the redshift, and the radio-loudness.

5. Spectral fits in the $0.1-4.0 \mathrm{keV}$ energy range confirm the correlation between the X-ray slope in this band and the radioloudness, with the radio-loud quasars having flatter slopes than the radio-quiet ones.

6. Five out of six objects with detected soft excess emission are radio-quiet. The detection of soft excess emission in oneradio-loud object (3C 273) is probably due to its much higher $\mathrm{X}$-ray flux.

This work was partially supported by NASA contract NAS8-30751 (HEAO 2) and NASA grant NAG5-1201 (A. D. P.). A. C. acknowledges hospitality and support from Center for Astrophysics where part of this work has been done. The EXOSAT team and in particular Frank Haberl and Gianpiero Tagliaferri are also thanked for help with the EXOSAT data reduction.
Arnaud, K. A., et al. 1985, MNRAS, 217, 105

Avni, Y. 1976, ApJ, 210, 642

Bevington, P. R. 1969, Data Reduction and Error Analysis for the Physical

Sciences (New York: McGraw-Hill)

Canizares, C. R., \& White, J. L. 1989, ApJ, 339, 27

De Korte, P. A. J., et al. 1981, Space Sci. Rev., 30, 495

Elvis, M., Green, R. F., Bechtold, J., Schmidt, M., Neugebauer, G., Soifer, B. T., Matthews, K, \& Fabbiano, G. 1986, ApJ, 310, 291

Elvis, M., Lockman, F. J., \& Wilkes, B. J. 1989, AJ, 97, 777

Elvis, M., et al. 1991, in preparation

Hasinger, G., Schmidt, M., \& Truemper, J. 1991, A\&A, 246, L2

Kellerman, K. I., Sramek, R., Schmidt, M., Shaffer, D. B., \& Green, R. F. 1989, AJ, 98, 1195

Kruper, J. S., Urry, C. M., \& Canizares, C. R. 1990, ApJS, 74, 347

Lampton, M., Margon, B., \& Bowyer, S. 1976, ApJ, 208, 177

Lawrence, A., \& Elvis, M. 1982, ApJ, 256, 410

Masnou, J. L., Wilkes, B. J., Elvis, M., McDowell, J. C., \& Arnaud, K. A. 1991, A\&A, in press

Matsuoka, M., Piro, L., Yamauchi, M., \& Murakami, T. 1990, ApJ, 361, 440

Morrison, R., \& McCammon, D. 1983, ApJ, 270, 119

Mushotzky, R. F. 1984, Adv. Space Res., 3, 157

Piro, L., Matsuoka, M., \& Yamauchi, M. 1989, in Proc. 23rd ESLAB Symposium on Two Topics in X-Ray Astronomy (ESA SP-296), ed. J. Hunt \& B. Battrick (Noordwijk: ESA), 819

Pounds, K. A., Nandra, K., Stewart, G. C., George, I. M., \& Fabian, A. C. 1990

Nature, 344,132

Reichert, G. A., Mushotzky, R. F., Petre, R., \& Holt, S. S. 1985, ApJ, 296, 69

\section{REFERENCES}

Schmidt, M., \& Green, R. F. 1983, ApJ, 269, 352

Setti, G. 1991, in Proc. Yamada Conference on "Frontiers of X-ray Astronomy," ed. Y. Tanaka \& K. Koyama (Tokyo: Universal Academy Press), in press

Shafer, R. A., Haberl, F., \& Arnaud, K. A. 1989, XSPEC User's Guide (ESA publication)

Siegel, S., \& Castellan, N. J. 1988, Nonparametric Statistics for the Behavioral Sciences (2d ed.; New York: McGraw-Hill)

Tananbaum, H., Avni, Y., Green, R. F., Schmidt, M., \& Zamorani, G. 1986, ApJ, 305, 57

Treves, A., Bouchet, P., Chiappetti, L., Ciapi, A., Falomo, R., Maraschi, L., \& Tanzi, G. 1988, ApJ, 330, 178

Turner, M. J. L., Smith, A., \& Zimmerman, H. V. 1981, Space Sci. Rev., 30, 513

Turner, M. J. L., et al. 1989, in Proc. 23rd ESLAB Symposium on Two Topics in X-Ray Astronomy (ESA SP-296), ed. J. Hunt \& B. Battrick (Noordwijk ESA), 769

Turner, T. J., \& Pounds, K. A. 1989, MNRAS, 240, 833 (TP89)

Urry, C. M., Arnaud, K. A., Edelson, R. A., Kruper, J. S., \& Mushotzky, R. F. 1989, in Proc. 23rd ESLAB Symposium on Two Topics in X-Ray Astronomy (ESA SP-296), ed. J. Hunt \& B. Battrick (Noordwijk: ESA), 789

White, N. E., \& Peacock, A. 1988, Mem. Soc. Astron. Ital., 59, 7

Wilkes, B. J., \& Elvis, M: 1987, ApJ, 323, 243 (WE87)

Williams, O. R., et al. 1991, ApJ, submitted

Zamorani, G. 1984, in Proc. Internat. Symp. X-Ray Astronomy '84, ed M. Oda \& R. Giacconi (Tokyo: Institute of Space and Astronautical Science), 419

Zamorani, G., et al. 1981, ApJ, 245, 357 